\title{
Error analysis of numerical Weyl fractional derivatives in the case of certain Hölder continuous functions
}

\author{
Juhani Nissilä \\ Mechatronics and Machine Diagnostics \\ Faculty of Technology, University of Oulu \\ Oulu, Finland \\ juhani.nissila@oulu.fi
}

\begin{abstract}
The calculation of fractional or integer order derivatives and integrals has been demonstrated to be simple and fast in the frequency domain. It is also the most sensible method if one wishes to calculate derivatives or integrals of periodic signals. In this paper, error analysis is carried out for the numerical algorithm for Weyl fractional derivatives. To derive an upper bound for the numerical error, some knowledge of the smoothness of the signal must be known in advance or it must be estimated. The derived error analysis is tested with sampled functions with known regularity and with real vibration measurements from rotating machines. Compared to previous publications which deal with error analysis of integer order numerical derivatives in the frequency domain using $L^{2}$ errors, the result of this paper is in terms of maximum absolute error and it is based on a novel result on the signal's regularity. The general conclusion using either error estimates is the same: the error of numerical Weyl derivatives is bounded by some constant times the sequence length raised to a negative power. The exponent depends on the smoothness of the signal. This contrasts with using difference quotients in numerical differentiation, in which case the error is bounded by a constant times the sequence length raised to a some fixed negative power and the order of the method defines that exponent.

Index Terms-fractional calculus, Weyl fractional derivatives, Fourier analysis, Hölder regularity, numerical error analysis
\end{abstract}

\section{INTRODUCTION}

The goal of this paper is to derive estimates for the numerical error of Weyl fractional derivatives calculated with the discrete Fourier transform. Using knowledge on the smoothness of the signal it is possible to state this error estimate in terms of maximum absolute error.

In Section II the mathematical definitions for smoothness of signals and Fourier analysis are presented. Section III is devoted to fractional calculus using Fourier series. The actual error estimates are derived in Section IV. The results are tested on signals with known regularity and also on real vibration measurements from a machine diagnostics test rig in Section $\mathrm{V}$ and finally the results are discussed in Section VI.

The author is currently doing research under a grant from the Finnish Cultural Foundation, North Ostrobothnia Regional Fund. This work has also been supported by the Riitta and Jorma J. Takanen foundation, Tauno Tönning foundation and Otto A. Malm foundation.

\section{Definitions}

Definition 1. The space $L^{p}(0, T), p \geq 1$ consists of those functions, for which the following norm is finite

$$
\|f\|_{p}=\left(\int_{0}^{T}|f(t)|^{p} \mathrm{~d} t\right)^{\frac{1}{p}} .
$$

Definition 2. Function $f:[0, T] \rightarrow \mathbb{C}$ is uniformly Hölder continuous of order $\mu=(0,1]$, if

$$
|f(t+h)-f(t)| \leq C h^{\mu}
$$

holds for all $t, t+h \in[0, T]$ and for all $h$ sufficiently small. Then we write $f \in C_{\mu}[0, T]$. If for some $m=1,2,3, \ldots$ it holds that $f^{(m)} \in C_{\mu}[0, T]$, we write $f \in C_{m, \mu}[0, T]$.

Definition 3. Function $f:[0, T] \rightarrow \mathbb{C}$ is absolutely continuous, i.e. $f \in A C[0, T]$, if its derivative $f^{\prime}$ exists a.e. on $[0, T]$, $f^{\prime} \in L^{1}(0, T)$ and

$$
\int_{0}^{t} f^{\prime}(\tau) \mathrm{d} \tau=f(t)-f(0) \quad \text { for every } t \in[0, T] .
$$

If $f^{(m)} \in A C[0, T]$ for some $m=0,1,2, \ldots$, then $f$ belongs to Sobolev space $W_{m+1}^{1}[0, T]$.

Definition 4. Let $f \in L^{1}(0, T)$ and $T$-periodic. Its Fourier series is

$$
\sum_{k=-\infty}^{\infty} c_{k}(f) e^{i 2 \pi k t / T},
$$

where the Fourier coefficients $c_{k}(f)$ are calculated as

$$
c_{k}(f)=\frac{1}{T} \int_{0}^{T} f(t) e^{-i 2 \pi k t / T} \mathrm{~d} t, \quad k=0, \pm 1, \pm 2, \ldots
$$

Definition 5. The discrete Fourier transform (DFT) of a sequence $\mathbf{f}=\left(f_{0}, \ldots, f_{N-1}\right) \in \mathbb{C}^{N}$ at the point $k$ is

$$
\mathcal{F}\{\mathbf{f}\}_{k}=F_{k}=\frac{1}{N} \sum_{n=0}^{N-1} f_{n} e^{-i 2 \pi k n / N}
$$


and its inverse transform (IDFT) at the point $n$ is

$$
\mathcal{F}^{-1}\{\mathbf{F}\}_{n}=f_{n}=\sum_{k=0}^{N-1} F_{k} e^{i 2 \pi k n / N} .
$$

The IDFT always returns the original sequence, and thus we have equated $\mathcal{F}^{-1}\{\mathbf{F}\}_{n}=f_{n}$ in the definition above [1, p. 80], [2, p. 30].

Definition 6. The forward difference operator $\Delta$ acts on a function $f$ with a difference interval $h>0$

$$
\Delta_{h} f(t)=f(t+h)-f(t) .
$$

Definition 7. The Sobolev space $H^{s}(0, T)$ of order $s>0$ consists of those functions, for which the following norm is finite

$$
\|f\|_{H^{s}}=\left(\sum_{k=-\infty}^{\infty}(1+|k|)^{2 s}\left|c_{k}(f)\right|^{2}\right)^{\frac{1}{2}}
$$

\section{FRACTIONAL CALCULUS}

Fractional calculus for periodic functions using Fourier series was first studied by Hermann Weyl in 1917 [3]. His first definition was for fractional integration of 1-periodic functions. A more general definition is as follows.

Definition 8. Let $c_{k}(f)$ be the Fourier coefficients of a $T$ periodic function or distribution $f$. Then the Weyl differintegral operator is

$$
{ }^{W} D^{z} f(t)=\sum_{k=-\infty}^{\infty}\left(\frac{i 2 \pi k}{T}\right)^{z} c_{k}(f) e^{i 2 \pi k t / T},
$$

where $(i 2 \pi k / T)^{z}=e^{z \ln (2 \pi|k| / T)+i z \frac{\pi}{2} \operatorname{sign}(k)}$ and $z=\alpha+i \beta$ for some $\alpha, \beta \in \mathbb{R}$.

Fractional differentiation decreases the uniform Höldercontinuity of a function and similarly fractional integration increases it.

Theorem 9. Let $0<\alpha<\mu<1$ and $f \in C_{\mu}[0, T]$. Then ${ }^{W} D^{\alpha} f \in C_{\mu-\alpha}[0, T]$.

Theorem 10. Let $0<\mu<1, \alpha<0$ and $f \in C_{\mu}[0, T]$. Then ${ }^{W} D^{\alpha} f \in C_{\mu-\alpha}[0, T]$, if $\mu-\alpha<1$.

Proof. Parts of Theorems 8.13 and 8.14 in [4, pp. 136-137].

The DFT and IDFT pair can be used for differentiation and integration of signals. An algorithm for this consists of calculating the DFT coefficients $F_{k}$ and then forming a new sequence $\mathbf{G}=\left(G_{0}, \ldots, G_{N-1}\right)$, with $G_{0}=0$ and

$$
\begin{aligned}
G_{k} & =\left(\frac{2 \pi k i}{T}\right)^{z} F_{k}, \quad 0<k<N / 2 \\
G_{N+k} & =\left(\frac{2 \pi k i}{T}\right)^{z} F_{N+k}, \quad-N / 2<k<0 \\
G_{N / 2} & =\left(\frac{\pi N}{T}\right)^{z} \cos \left(z \frac{\pi}{2}\right) F_{N / 2} \quad \text { (if } N \text { is even). }
\end{aligned}
$$

Finally we get the estimate for the differintegral as vector $\tilde{\mathbf{f}}^{(z)}$ with the IDFT

$$
\tilde{f}_{n}^{(z)}=\mathcal{F}^{-1}\{\mathbf{G}\}_{n} .
$$

The only problematic part in deriving this algorithm is the term $G_{N / 2}$ for even $N$, which the author has presented in [5]. The algorithm works with complex $z$, in which case the values of $\left(\frac{2 \pi k i}{T}\right)^{z}$ and $\left(\frac{\pi N}{T}\right)^{z}$ are computed similarly as in the definition of the Weyl differintegral operator.

The components $F_{k}$ of the DFT are intimately related to the Fourier coefficients $c_{k}(f)$ of the original function.

Lemma 11. If the Fourier coefficients of the periodic function $f$ are absolutely summable, i.e. $c_{k}(f) \in l^{1}(\mathbb{Z})$, then

$$
F_{k}=\sum_{l=-\infty}^{\infty} c_{k+l N}(f) \text {. }
$$

Proof. [1, pp. $78-79]$

The equation (13) is called the Discrete Poisson summation formula in [2, p. 182]. If the signal is band-limited, i.e. its Fourier coefficients are zero for all $|k|>M$, then the previous Lemma tells us that with high enough sample number $N$ we can deduce the exact Fourier coefficients with the DFT. A special case appears if we use exactly $M=N / 2$ terms (this is also discussed in [2, p. 182]), in which case

$$
\begin{aligned}
F_{k} & =c_{k}(f), \quad 0 \leq k<N / 2 \\
F_{N+k} & =c_{k}(f), \quad-N / 2<k<0 \\
F_{N / 2} & =c_{-N / 2}(f)+c_{N / 2}(f)=2 \operatorname{Re}\left\{c_{N / 2}(f)\right\},
\end{aligned}
$$

and in the last step we presumed that $f$ is real-valued, in which case the corresponding positive and negative Fourier coefficients are complex conjugates. This special type of aliasing is the reason why the term $F_{N / 2}$ was handled specially in (11).

\section{ERROR ESTIMATION}

Let us first note that in deriving the algorithm (11) a trigonometric interpolation between the discrete points of the estimated fractional derivative was presented in [5] as

$$
\begin{aligned}
\tilde{f}^{(z)}(t)= & \sum_{0 \leq k<N / 2} G_{k} e^{i 2 \pi k t / T}+\sum_{-N / 2<k<0} G_{N+k} e^{i 2 \pi k t / T} \\
& +\left(\frac{\pi N}{T}\right)^{z} F_{N / 2} \cos \left(\frac{\pi N t}{T}+z \frac{\pi}{2}\right),
\end{aligned}
$$

where the term $F_{N / 2}$ is of course included only if $N$ is even.

In [6] it has been proved that for a function $f \in H^{s}(0, T)$, $s>1$, the $L^{2}$ error of the numerical first derivative with the Fourier method is

$$
\left\|D^{1} f(t)-\tilde{f}^{(1)}(t)\right\|_{2} \leq \frac{E\|f\|_{H^{s}}}{N^{s-1}},
$$

although the term $F_{N / 2}$ is neqlected in the analysis (as it very often is). The effect of one term can be included in the constant $E$. This error combines the effects of aliasing and truncation. Similar analysis is done for the Fourier transform 
and functions in the Sobolev space defined on the real line in [7]. We would like to form a similar result for the maximum absolute error for periodic functions and also derive useful constants to calculate the actual error estimates. To this end we need to estimate infinite sums with integrals.

Lemma 12 (Integral test of convergence). Let $f$ be a positive and decreasing function on the interval $[M, \infty)$. Then the series $\sum_{l=M}^{\infty} f(l)$ converges if and only if the integral $\int_{M}^{\infty} f(t) \mathrm{d} t$ converges and then we have the estimates

$$
\int_{M}^{\infty} f(t) \mathrm{d} t \leq \sum_{l=M}^{\infty} f(l) \leq f(M)+\int_{M}^{\infty} f(t) \mathrm{d} t .
$$

Proof. [8, p. 435]

We also utilise the following novel result by the author in the error analysis.

Theorem 13. Suppose that $f \in W_{m+1}^{1}[0, T] \cap C_{m, \mu}[0, T]$ with some $\mu \in(0,1], f$ is $T$-periodic, and the number of maxima and minima of the function $\Delta_{h} f^{(m)}$ is uniformly bounded for every $0<h \leq h_{0}$. Then the Fourier coefficients of $f$ decay like $c_{k}(f) \in O\left(1 /|k|^{1+m+\mu}\right)$ as $|k| \rightarrow \infty$.

Proof. [9]

It is also proved in [9] that the decay rate $c_{k}(f) \in$ $O\left(1 /|k|^{1+m+\mu}\right)$ implies the same uniform Hölder continuity (thus Theorems 9 and 10 are obvious for these special functions), but absolute continuity and the finite oscillations condition may not be read from the decay rate. Thus if we want to use Theorem 13 in estimating smoothness, some assumptions about the signals must be made..

So let us suppose that the original analog signal $f$ is in $C_{m, \mu}[0, T]$ and that $f^{(m)}$ is absolutely continuous and $\Delta_{h} f^{(m)}$ does not oscillate infinitely often. Then Theorem 13 tells us that $\left|c_{k}(f)\right| \leq B /|k|^{1+m+\mu}$. Thus the spectrum of the signal is infinite in length. In physical measurements, it is quite typical to use an anti-aliasing filter before data is stored in digital form. So next our analog signal goes through an antialiasing filter and is then sampled into a sequence $\mathbf{f}$. The analog filtering can effectively guarantee that we can compute the $M$ first correct Fourier coefficients with the DFT.

Plotting the amplitude spectrum on a log-log-scale we can estimate the value $1+m+\mu$ from the slope of the decay. The value of the fitted line at 0 gives an estimate for the constant $\log (B)$ in the inequality

$$
\log \left|c_{k}(f)\right| \leq \log (B)-(1+m+\mu) \log |k| .
$$

Finally we use the DFT to compute fractional derivatives or integrals of $f$ with (12). Now we can bound the absolute error of this numerical differintegral $\tilde{f}_{n}^{(z)}$. For simplicity let us first assume that $N$ is odd, $z=\alpha$ real-valued and since there is no aliasing we get

$$
\begin{aligned}
& \left|{ }^{W} D^{z} f(t)-\tilde{f}^{(z)}(t)\right| \\
& =\left|\sum_{|k| \geq N / 2}\left(\frac{i 2 \pi k}{T}\right)^{z} c_{k}(f) e^{i 2 \pi k t / T}\right| \\
& \leq \sum_{|k| \geq N / 2}\left|\left(\frac{i 2 \pi k}{T}\right)^{z}\right|\left|c_{k}(f)\right| \\
& \leq\left|\left(\frac{2 \pi}{T}\right)^{z}\right| \sum_{|k| \geq N / 2} \frac{|k|^{z} B}{|k|^{1+m+\mu}} \\
& =2 B\left|\left(\frac{2 \pi}{T}\right)^{z}\right| \sum_{k \geq N / 2} \frac{1}{k^{1+m+\mu-z}} \\
& \leq 2 B\left|\left(\frac{2 \pi}{T}\right)^{z}\right|\left(\frac{1}{\left\lceil\frac{N}{2}\right\rceil^{1+m+\mu-z}}+\int_{\left\lceil\frac{N}{2}\right\rceil}^{\infty} \frac{\mathrm{d} \nu}{\nu^{1+m+\mu-z}}\right) \\
& =\left|\left(\frac{2 \pi}{T}\right)^{z}\right| \frac{2 B}{\left\lceil\frac{N}{2}\right\rceil^{m+\mu-z}}\left(\frac{1}{\left\lceil\frac{N}{2}\right\rceil}+\frac{1}{m+\mu-z}\right),
\end{aligned}
$$

where $\left\lceil\frac{N}{2}\right\rceil$ is the next largest integer to $N / 2$.

Now let us suppose that $N$ is even and the only aliasing happens at the highest frequency according to (14). Then $c_{N / 2}(f)$ and $c_{-N / 2}(f)$ are complex conjugates for real-valued $f$ and the sum of the highest positive and negative frequencies in the Fourier series is

$$
\begin{aligned}
& \left(\frac{i \pi N}{T}\right)^{z} c_{\frac{N}{2}}(f) e^{i \pi N t / T}+\left(\frac{-i \pi N}{T}\right)^{z} c_{-\frac{N}{2}}(f) e^{-i \pi N t / T} \\
& =\left(\frac{\pi N}{T}\right)^{z} 2 \operatorname{Re}\left\{c_{N / 2}(f)\right\} \cos \left(\frac{\pi N t}{T}+z \frac{\pi}{2}\right) \\
& \quad+i\left(\frac{\pi N}{T}\right)^{z} 2 \operatorname{Im}\left\{c_{N / 2}(f)\right\} \sin \left(\frac{\pi N t}{T}+z \frac{\pi}{2}\right) .
\end{aligned}
$$

Now we may check whether the term $F_{N / 2}$ has any effect on our estimate

$$
\begin{aligned}
\mid W & D^{z} f(t)-\tilde{f}^{(z)}(t) \mid \\
= & \mid \sum_{|k| \geq N / 2}\left(\frac{i 2 \pi k}{T}\right)^{z} c_{k}(f) e^{i 2 \pi k t / T} \\
& -\left(\frac{\pi N}{T}\right)^{z} F_{\frac{N}{2}} \cos \left(\frac{\pi N t}{T}+z \frac{\pi}{2}\right) \mid \\
= & \mid \sum_{|k|>N / 2}\left(\frac{i 2 \pi k}{T}\right)^{z} c_{k}(f) e^{i 2 \pi k t / T} \\
& +i\left(\frac{\pi N}{T}\right)^{z} 2 \operatorname{Im}\left\{c_{\frac{N}{2}}(f)\right\} \sin \left(\frac{\pi N t}{T}+z \frac{\pi}{2}\right) \mid \\
\leq & \sum_{|k|>N / 2}\left|\left(\frac{i 2 \pi k}{T}\right)^{z}\right|\left|c_{k}(f)\right|+\left|\left(\frac{\pi N}{T}\right)^{z}\right| 2\left|\operatorname{Im}\left\{c_{\frac{N}{2}}(f)\right\}\right| \\
\leq & \sum_{|k| \geq N / 2}\left|\left(\frac{i 2 \pi k}{T}\right)^{z}\right|\left|c_{k}(f)\right| .
\end{aligned}
$$

The cosine term corresponding to the highest frequency was thus eliminated, but since it is impossible to get information 
on $\operatorname{Im}\left\{c_{N / 2}(f)\right\}$ with the DFT, we estimated up by adding the cosine term back. The final result will be the same (19) as with the odd $N$, the only difference being that $\left\lceil\frac{N}{2}\right\rceil=\frac{N}{2}$.

Notice that the upper bound on the error is the same at all points $t$. The actual error of course will vary between points, but the uniform bound is a result of our simple estimation of the Fourier series representation.

If aliasing according to (13) is present, then the trigonometric interpolant (15) for the estimate of the Weyl derivative becomes for odd $N$

$$
\begin{aligned}
\tilde{f}^{(z)}(t)= & \sum_{|k|<N / 2}\left(\frac{i 2 \pi k}{T}\right)^{z} c_{k} e^{i 2 \pi k t / T} \\
& +\sum_{|k|<N / 2}\left(\sum_{l \neq 0}\left(\frac{i 2 \pi k}{T}\right)^{z} c_{k+l N}(f)\right) e^{i 2 \pi k t / T} .
\end{aligned}
$$

Thus we must also estimate the sum

$$
\begin{aligned}
& \left|\sum_{|k|<N / 2}\left(\sum_{l \neq 0}\left(\frac{i 2 \pi k}{T}\right)^{z} c_{k+l N}(f)\right)\right| \\
& \leq\left|\left(\frac{2 \pi}{T}\right)^{z}\right| \sum_{|k|<N / 2}\left(\sum_{l \neq 0} \frac{|k|^{z} B}{|k+l N|^{1+m+\mu}}\right) \\
& =\left|\left(\frac{2 \pi}{T}\right)^{z}\right| \sum_{|k|<N / 2}\left(\sum_{l=1}^{\infty} \frac{|k|^{z} 2 B}{|k+l N|^{1+m+\mu}}\right) \\
& \leq\left|\left(\frac{2 \pi}{T}\right)^{z}\right| \sum_{|k|<N / 2}\left(\sum_{l=1}^{\infty} \frac{|k|^{z} 2 B}{\left(l N-\frac{N}{2}\right)^{1+m+\mu}}\right) \\
& =\left|\left(\frac{2 \pi}{T}\right)^{z}\right| \sum_{|k|<N / 2} \frac{|k|^{z} 2 B}{N^{1+m+\mu}\left(\sum_{l=1}^{\infty} \frac{1}{\left(l-\frac{1}{2}\right)^{1+m+\mu}}\right)} \\
& \leq\left|\left(\frac{2 \pi}{T}\right)^{z}\right| \frac{N \cdot\left(\frac{N}{2}\right)^{z} 2 B}{N^{1+m+\mu}}\left(2^{1+m+\mu}+\int_{\frac{1}{2}}^{\infty} \frac{\mathrm{d} \nu}{\nu^{1+m+\mu}}\right) \\
& =\left|\left(\frac{\pi}{T}\right)^{z}\right| \frac{2 B}{N^{m+\mu-z}}\left(2^{1+m+\mu}+\frac{2^{m+\mu}}{m+\mu}\right)^{\frac{2 B}{m+m}}
\end{aligned}
$$

If $N$ is even, we will also have the aliasing series from the term $F_{N / 2}$ of (15) but to simplify the error estimate, this is estimated upwards (note that the effect of $c_{-N / 2}(f)$ was already considered earlier)

$$
\begin{aligned}
& \left|\sum_{|k|<N / 2}\left(\sum_{l \neq 0}\left(\frac{i 2 \pi k}{T}\right)^{z} c_{k+l N}(f)\right)\right| \\
& +\left|\sum_{l \geq 1, l \leq-2}\left(\frac{i \pi N}{T}\right)^{z} c_{\frac{N}{2}+l N}(f)\right| \\
& \leq \sum_{|k| \leq N / 2}\left|\left(\sum_{l \neq 0}\left(\frac{i 2 \pi k}{T}\right)^{z} c_{k+l N}(f)\right)\right|,
\end{aligned}
$$

and we see that bounding this will result to the same equation (20), since we bounded $|k|$ by $N / 2$ when deriving it.

Thus we have proved the following Theorem.
Theorem 14. If the Fourier coefficients of the periodic function $f$ decay like $c_{k}(f) \in O\left(1 /|k|^{1+m+\mu}\right)$ with some $m=0,1,2, \ldots$ and $\mu \in(0,1]$, then for $0<z<m+\mu$

$$
\max _{t \in[0, T]}\left|W D^{z} f(t)-\tilde{f}^{(z)}(t)\right| \leq \frac{U}{N^{m+\mu-z}},
$$

where $N$ is the number of discrete samples of the function on the interval $[0, T]$ and $U$ does not depend on $N$. This is a combination of truncation and aliasing errors (19) and (20).

\section{EXAMPLES}

First we study the convergence of the maximum absolute error in the case of the first derivative of a simple function $\cos (\pi t)$ on the interval $[0,7]$. The periodic continuation of this function is thus not continuous, since $\cos (0)=1 \neq-1=$ $\cos (7 \pi)$. Figure 1 shows the convergence of the maximum absolute error using different window functions as the number of sample points increase. The window function $w_{1}$ is the Tukey, or tapered cosine window

$$
w_{1}(t)= \begin{cases}0, & \text { if } t \leq 0 \\ \frac{1}{2}\left(1-\cos \left(\pi \frac{t}{T / \epsilon}\right)\right), & \text { if } 0<t<T / \epsilon \\ 1, & \text { if } T / \epsilon \leq t \leq T / 2 \\ w_{1}(T-t), & \text { if } t>T / 2,\end{cases}
$$

Application of $w_{1}$ gives the signal one continuous derivative at the end points. An infinitely smooth window function was defined in [10]

$$
w_{2}(t)= \begin{cases}0, & \text { if } t \leq 0 \\ \frac{1}{A} \int_{0}^{t} e^{(\tau(\tau-T / \epsilon))^{-1}} \mathrm{~d} \tau, & \text { if } 0<t<T / \epsilon \\ 1, & \text { if } T / \epsilon \leq t \leq T / 2 \\ w_{2}(T-t), & \text { if } t>T / 2\end{cases}
$$

where $A=\int_{0}^{T / \epsilon} e^{(\tau(\tau-T / \epsilon))^{-1}} \mathrm{~d} \tau$ and we use the value $\epsilon=10$. The maximum absolute error is calculated from the interval where the window is flat, i.e. $10 \%$ from both ends is not taken into account when using window functions and the whole interval is used when there is no window function. Values of the window function $w_{2}$ have been integrated numerically with the trapezoidal rule and the Simpson's rule, but these two perform almost identically in Fig. 1. Their convergence is likely exponential (i.e. faster than linear on the log-log scale) whereas with $w_{1}(22)$ the convergence is only polynomial (i.e. linear on the log-log scale) with slope -1 . This is exactly what equations (19) and (20) predict, since the signal is in $C_{1,1}[0,7]$ after application of $w_{1}$. Application of $w_{2}$ makes the signal infinitely smooth, so the equations (19) and (20) are valid for any $m \in \mathbb{N}$, although the constants are different for every $m$ as well (in [6] it is proved that the $L^{2}$ error decreases exponentially if the signal is analytic). With $w_{2}$ the minimum error $\left(\approx 10^{-12}\right)$ is reached when $N$ is roughly 1000 and with bigger $N$ the error actually diverges because we are so close to machine precision. Without any window function the maximum absolute error in Fig. 1 actually diverges with slope +1 . 


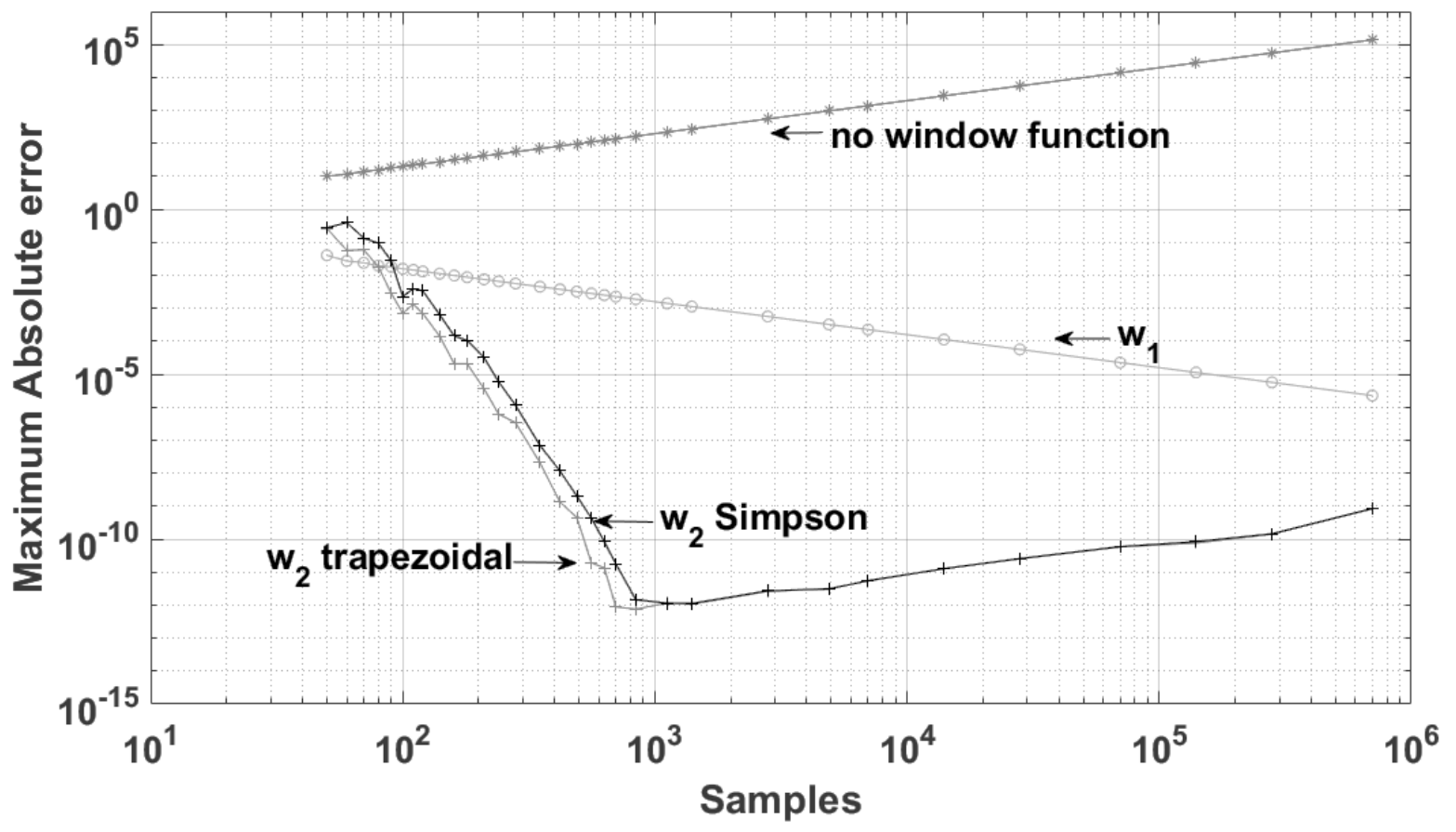

Fig. 1. Convergence of the maximum absolute error of the first derivative of $\cos (\pi t)$ on the interval $[0,7]$ as the number of sample points increase.

This same example was studied also in [5] but the corresponding error there was the local error near the center point of the interval, i.e. at $t=3.5$. Interestingly the local error decreased at a polynomial rate with slope -3 when using the Tukey window and even with slope -1 when using no window function at all. So it is possible that the local absolute error decreases with increasing sample number near the points where the function is smooth enough although the maximum absolute error increases at the same time! This is a clear demonstration of the local properties of the Fourier series representation even though it is difficult to read these local properties directly from the Fourier coefficients alone. In [11, p. 31] this is stated as the Riemann localisation principle: if the function $f \in L^{1}(0, T)$ is identically zero on an open interval, then on any of its compact subinterval the Fourier partial sums tend uniformly to zero as the number of terms in the partial sums tend to infinity.

Acceleration measurements from a vibration test rig were analysed to estimate their regularity. The measurements have been analysed before for example in [12] and [13] and the measurement setup is carefully explained in those papers as well. The two measurements used in this paper are measured axially from bearing 3 of the test rig when the frequency of rotation is $20 \mathrm{~Hz}$. The first measurement is performed when no fault is present and the second when lubrication is completely washed away from bearing 3. Sampling frequency is $50 \mathrm{kHz}$ and $12 \mathrm{~s}$ long measurements are analysed. The accelerometer has resonance frequency centered at $15 \mathrm{kHz}$ and thus only frequencies up to $10 \mathrm{kHz}$ are displayed in Fig. 2 and 3.
The frequencies seem to decrease in the range $1 \mathrm{kHz}-10$ $\mathrm{kHz}$ and linear lines were fitted above this region. From Fig. 2 we estimate the slope of the line when the bearing is healthy

$$
\frac{\log (0.174)-\log (0.001178)}{\log (1136)-\log (8978)} \approx-2.416
$$

and from Fig. 3 we estimate the slope of the line when the bearing is dry

$$
\frac{\log (1.161)-\log (0.1588)}{\log (2646)-\log (8667)} \approx-1.677 .
$$

The vibration has thus become more irregular when the fault is present. When the bearing is healthy, according to the error analysis of this paper the acceleration measurement can be differentiated with order $z=1.416$ before the error bound diverges. When the bearing is dry, only fractional derivative of order $z=0.677$ is required for the error bound to diverge.

\section{DISCUSSION}

Bounds for the maximum absolute error of numerical Weyl fractional derivatives were carried out for certain Hölder continuous functions whose Fourier coefficients decay like $O\left(1 /|k|^{1+m+\mu}\right)$. The error bound (19) is caused by truncation of higher frequencies and (20) by aliasing. Both decay asymptotically as $O\left(1 / N^{m+\mu-z}\right)$, where $z$ is the order of the fractional derivative. The error bound diverges when the order of the derivative reaches $z=m+\mu$ and then the spectrum of the differentiated signal decays like $O(1 /|k|)$. This is the decay rate that discontinuous functions or more generally functions of bounded variation achieve. The actual error may probably 


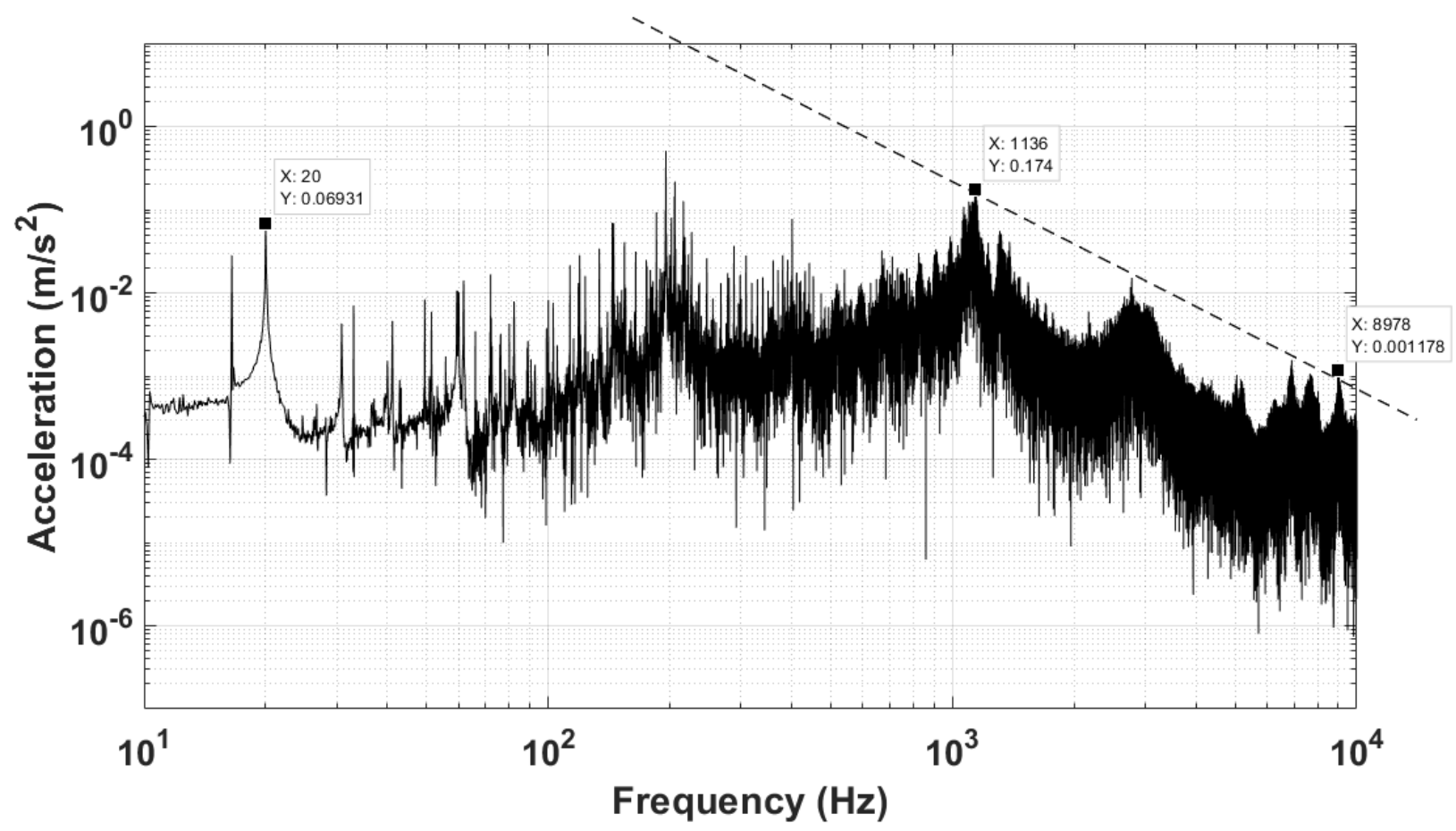

Fig. 2. Amplitude spectrum from the axial vibration from bearing 3 when no fault is present.

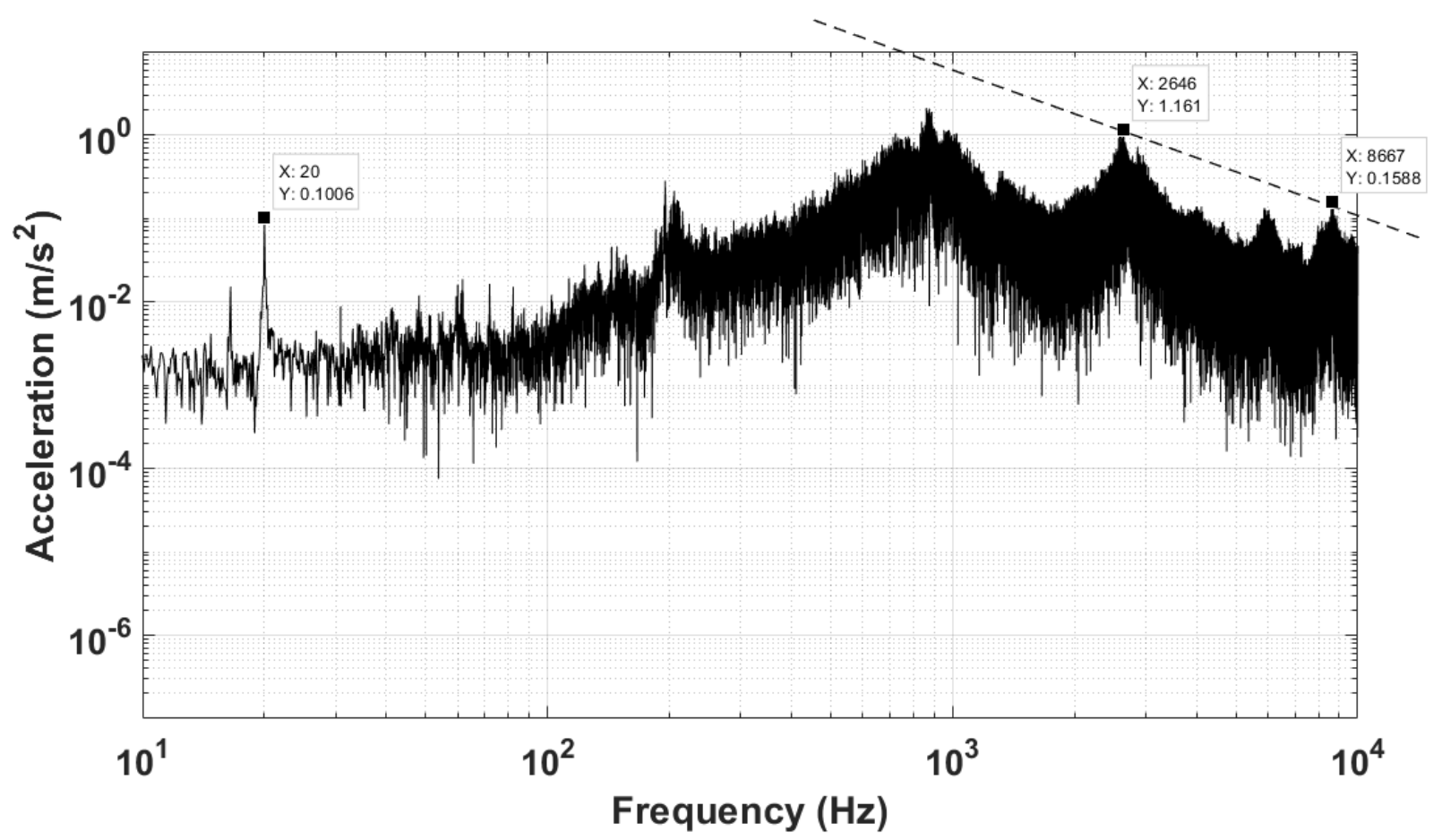

Fig. 3. Amplitude spectrum from the axial vibration from bearing 3 when the bearing is dry. 
still be small especially near the points where the signal is smoothest, but it is naturally hard to bound the error uniformly from this point on.

The estimates can be used in analysing real vibration measurements as well, although some assumptions about the smoothness must also be made beforehand.

\section{ACKNOWLEDGMENT}

I wish to thank Jouni Laurila for providing the vibration measurements that were utilised in this paper.

All web addresses retrieved on 08.05.2018.

\section{REFERENCES}

[1] V. Serov, Fourier series and Fourier transform and their applications in mathematical physics, 451 p., Oulu: University of Oulu, 2015.

[2] W. Briggs and V. E. Henson, The DFT an owner's manual for the discrete Fourier transform, 432 p., Philadelphia: SIAM, 1995

[3] H. Weyl, "Bemerkungen zum Begriff des Differentialquotienten gebrochener Ordnung," Vierteljahrsschrift der Naturforschenden Gesellschaft in Zürich, vol. 62, no. 1-2, pp. 296-302, 1917.

[4] A. Zygmund, Trigonometric series, volume 2, Second corrected edition, 364 p., Cambridge: Cambridge University press, 1968.

[5] J. Nissilä, S. Lahdelma and J. Laurila, "Condition monitoring of the front axle of a load haul dumper with real order derivatives and generalised norms," Proc. 11th Int. Conf. on Condition Monitoring and Machinery Failure Prevention Technologies, CM 2014/MFPT 2014, Manchester, UK, 2014.

https://www.researchgate.net/publication/286767140_Condition_ monitoring_of_the_front_axle_of_a_load_haul_dumper_with_real_ order_derivatives_and_generalised_norms

[6] E. Tadmor, "The exponential accuracy of Fourier and Tchebyshev differencing methods," SIAM J. Sci. Comput. vol. 23, no. 1, pp. 110, 1986.

https://ntrs.nasa.gov/archive/nasa/casi.ntrs.nasa.gov/19840025037.pdf

[7] Z. Qian, C.-L. Fu, X.-T. Xiong and T. Wei, "Fourier truncation method for high order numerical derivatives," Applied Mathematics and Computation, vol. 181, pp. 940-948, 2006. https://doi.org/10.1016/j.amc.2006.01.057

[8] G. E. Shilov, Elementary Real and Complex Analysis, Revised English edition, Translated and edited by R. A. Silverman, New York: Dover publications, 1996.

[9] J. Nissilä, "Fourier decay of absolutely and Hölder continuous functions with infinitely or finitely many oscillations," unpublished, preprint available at https://arxiv.org/abs/1805.02445

[10] S. Lahdelma and V. Kotila, "Complex derivative - a new signal processing method," Kunnossapito, vol. 19, no. 4, pp. 39-46, 2005 https://www.researchgate.net/publication/266266752_Complex _ Derivative - A N New_Signal_Processing_Method

[11] M. A. Pinsky, Introduction to Fourier Analysis and Wavelets, Pacific Grove: Brooks/Cole, 2002.

[12] S. Lahdelma, J. Laurila, J. Strackeljan and R. Hein "Separating different vibration sources in complex fault detection," Proc. 8th Int. Conf. on Condition Monitoring and Machinery Failure Prevention Technologies, CM 2011/MFPT 2011, Cardiff, UK, 2011.

[13] J. Laurila and S. Lahdelma "Advanced fault diagnosis by means of complex order derivatives," Insight Non-Destructive Testing and Condition Monitoring, vol. 56, no. 8, pp. 439-442, 2014.

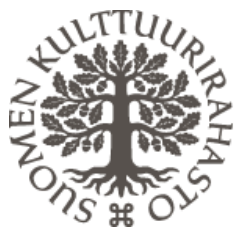

\section{Finnish Cultural Foundation}

